\title{
A Novel Approach to the Synthesis of N-Substituted 1-C-Aminomethyl Glycofuranosides
}

\author{
Yolanda Vera-Ayoso, ${ }^{\mathrm{a}}$ Pastora Borrachero, ${ }^{\mathrm{a}}$ Francisca Cabrera-Escribano, ${ }^{* \mathrm{a}}$ Manuel Gómez-Guillén, ${ }^{\mathrm{a}}$ Pierre Vogel ${ }^{\mathrm{b}}$ \\ a Departamento de Química Orgánica 'Profesor García González', Facultad de Química, Universidad de Sevilla, \\ Apartado de Correos No. 553, 41071 Sevilla, Spain \\ Fax +34(95)4624960; E-mail: fcabrera@us.es \\ b Laboratoire de Glycochimie et Synthèse Asymétrique, Swiss Federal Institute of Technology (EPFL), \\ BCH-EPFL, 1015 Lausanne, Switzerland \\ Received 18 October 2005
}

\begin{abstract}
Reductive amination of formyl $C$-glycofuranosides, easily available from hexose-derived equatorial-2-OH-glycopyranosides by DAST-promoted ring contraction, afforded N-substituted $1-C$-aminomethyl glycofuranosides in most cases in high yields.
\end{abstract}

Key words: $1-C$-aminomethyl glycofuranosides, formyl $C$-glycofuranosides, reductive amination, DAST-promoted ring contraction, sugar diamines

The stereoselective synthesis of functionalized $C$-glycosides has become an important area of carbohydrate research, as many naturally occurring $C$-glycosides show useful antibacterial, antiviral, and antitumoral properties. ${ }^{1}$ One significant type of $C$-glycoside derivatives are 1-Caminomethyl glycosides. These compounds are key intermediates for glycoconjugate syntheses, and a number of them have proved to be glycosidase inhibitors. ${ }^{2}$ Sugar amino acids $\mathbf{2}^{3}$ and $\mathbf{3},{ }^{4}$ which containing the substructure 1 (Figure 1), are dipeptide isosters and have been used as secondary structure inducing elements for the generation of peptide-based drugs. This kind of substructure is also found in the naturally occurring alkaloid muscarine (4), a rigid muscarinic agonist of acetylcholine, ${ }^{5}$ for which a renewed interest is due, in part, to the suggestion that various subtypes of muscarinic receptors seem to be implicated $^{6}$ in Alzheimer's disease.

Syntheses of 1-C-aminomethyl glycosides so far described rely on the introduction of a $\mathrm{CH}_{2} \mathrm{NH}_{2}$ equivalent at the anomeric position: (a) as $\mathrm{CH}_{3} \mathrm{NO}_{2}$ via nucleophilic aldol reaction, ${ }^{7}$ (b) by reducing the corresponding glyco-
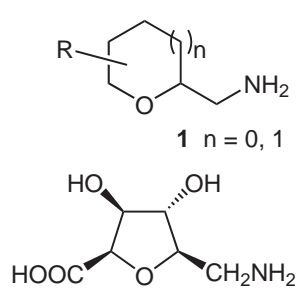

3

Figure 1

SYNLETT 2006, No. 1, pp 0045-0048

Advanced online publication: 16.12.2005

DOI: 10.1055/s-2005-922762; Art ID: D32105ST

(c) Georg Thieme Verlag Stuttgart · New York syl cyanide ${ }^{8}$ or, (c) by degradation of a $C$-vinyl glycoside and subsequent formation of the azidomethyl intermediate. ${ }^{3 \mathrm{~b}}$ Alternatively, rearrangement involving 5-exo $\mathrm{S}_{\mathrm{N}} 2$ opening of a terminal aziridine ring, and transformation of a primary hydroxyl function into the corresponding azide gave access to the 2,5-anhydro derivatives $3^{4}$ and 4,9 respectively. These routes are rather complicated, and for those involving an anomeric carbon-carbon bond-forming reaction, chemical efficiency and stereocontrol remain a difficult task. Moreover, for reaching an N-substituted 1-C-aminomethyl glycoside, additional $\mathrm{N}$-alkylation process would be still required.

Here we introduce a straightforward approach to N-substituted 1-C-aminomethyl glycofuranosides from hexosederived equatorial-2-OH-glycopyranosides (Scheme 1). The strategy takes advantage of a diethylaminosulfur trifluoride (DAST)-promoted ring contraction that, under remarkably mild conditions, leads to formyl $C$-glycofuranosides. ${ }^{10}$ The use of these compounds in standard coupling reactions with nucleophiles should provide a ready access to hydrolytically stable $C$-glycofuranosidebased molecules ( $C$-oligosaccharides and $C$-glycoconjugates). With this aim, our first goal has been to explore their coupling with biologically relevant amines as nitrogen-containing nucleophiles.

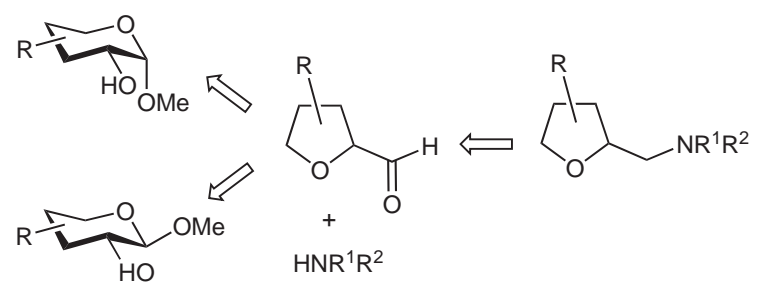

Scheme 1

We describe herein the synthesis of enantiopure orthogonally protected $C$-glycofuranosyl diamines by reductive amination of formyl $C$-glycofuranosides, easily obtained as their synthetic equivalents $\mathbf{6}$ and $\mathbf{1 2}$ from the methyl equatorial-2-OH-glycohexopyranosides $\mathbf{5}$ and 11, respectively, by DAST methodology. ${ }^{10,11}$

Treatment of the crude aldehyde obtained in situ by hydrolysis (9:1 TFA- $\mathrm{H}_{2} \mathrm{O}$, r.t., $1 \mathrm{~h}$ ) of the dimethyl acetal 6 , with diverse primary or secondary amines (1.4 mol equiv) 


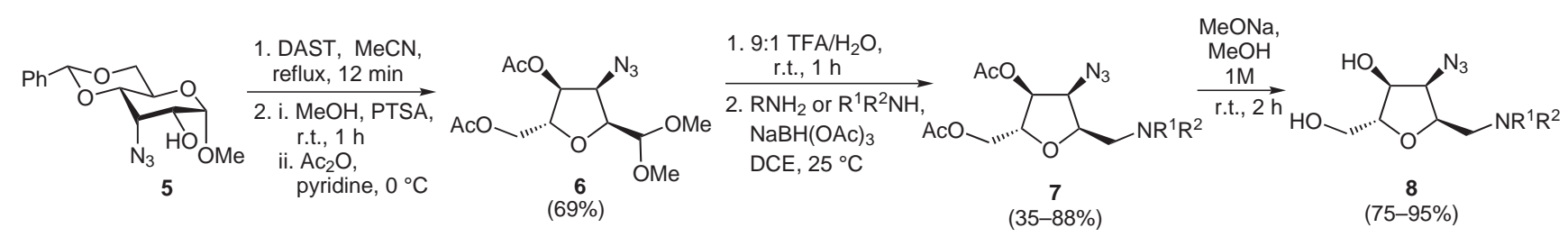

Scheme 2

in dry 1,2-dichloroethane, and subsequent reduction of the respective, not isolated, imine using sodium triacetoxyborohydride (1.4 mol equiv), afforded the respective compounds $\mathbf{7 a}-\mathbf{h}$ in moderate to high yields (Scheme 2, Table 1). ${ }^{12}$ Their deprotection with $1 \mathrm{M} \mathrm{NaMeO}-\mathrm{MeOH}$ gave the corresponding products $\mathbf{8 a}-\mathbf{h} .^{13}$

As shown in Table 1, primary and secondary aliphatic amines (benzylamine, piperidine, benzyloxycarbonyl piperazine, and morpholine, entries 1-4), as well as the aromatic amine 4-hydroxymethyl aniline (entry 6), gave the corresponding reductive amination compounds $\mathbf{7 a}-\mathbf{e}$ as the sole product. In the case of the other aniline derivatives (2-biphenylamino, ethyl 4-aminobenzoate and 4aminobenzonitrile, entries 8-10), however, reductive amination products $\mathbf{7 f}-\mathbf{h}$ were obtained together with the primary alcohol $\mathbf{1 0}$.

Starting from $N$-aminomorpholine as the amine (entry 5), the only isolated product was the hydrazone $\mathbf{9}$, which could not be reduced by the reagent employed. When using imidazole as the starting amine (entry 7), the expected

Table 1 Reductive Amination Products 7 of the Formyl Azido- $C$-glycofuranoside Synthetic Equivalent 6 with Various Amines, and Their Deacetylated Products $\mathbf{8}^{\mathrm{a}}$

\begin{tabular}{|c|c|c|c|c|}
\hline Entry & Amines $\mathrm{R}^{1} \mathrm{R}^{2} \mathrm{NH}$ & Reaction time (h) & Products $7^{12}$ (yield after purification, $\%$ ) & Products $\mathbf{8}^{13}$ (yield, \%) \\
\hline 1 & & 3 & $7 \mathbf{a}(55)$ & $\mathbf{8 a}(75)$ \\
\hline 2 & & 5 & $7 b(65)$ & $\mathbf{8 b}(92)$ \\
\hline 3 & & 2.5 & $7 \mathbf{c}(80)$ & $8 \mathbf{c}(95)$ \\
\hline 4 & & 6 & 7d (67) & $\mathbf{8 d}(87)$ \\
\hline 5 & & 1.5 & $\begin{array}{l}\mathrm{AcO} \backslash \\
\mathbf{9}(88)\end{array}$ & $-^{\mathrm{b}}$ \\
\hline 6 & & 2 & $7 e(77)$ & $\mathbf{8 e}(90)$ \\
\hline 7 & & 20 & $\begin{array}{l}\mathrm{AcO} \backslash{ }_{1} \cdot \cdots \\
\mathbf{1 0}^{11}(56)\end{array}$ & - \\
\hline 8 & & 18 & $\mathbf{7 f}(63)+\mathbf{1 0}(19)$ & $8 f(85)$ \\
\hline 9 & & 18 & $7 g^{11}(47)+10(34)$ & $8 g(86)$ \\
\hline 10 & & 18 & $7 \mathrm{~h}(35)+10(48)$ & $\mathbf{8 h}(89)$ \\
\hline
\end{tabular}

\footnotetext{
${ }^{a}$ All products were fully characterized by their IR, ${ }^{1} \mathrm{H}$ NMR, ${ }^{13} \mathrm{C}$ NMR, and HRMS spectral data. ${ }^{14}$

${ }^{\mathrm{b}}$ Complex mixture of products.
} 


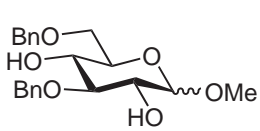

11

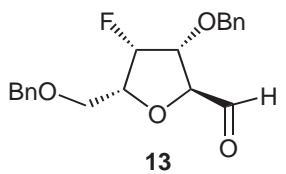

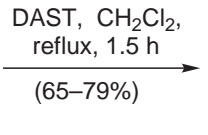

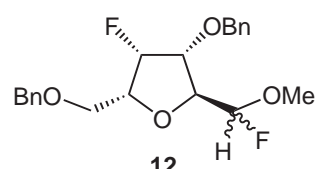

12
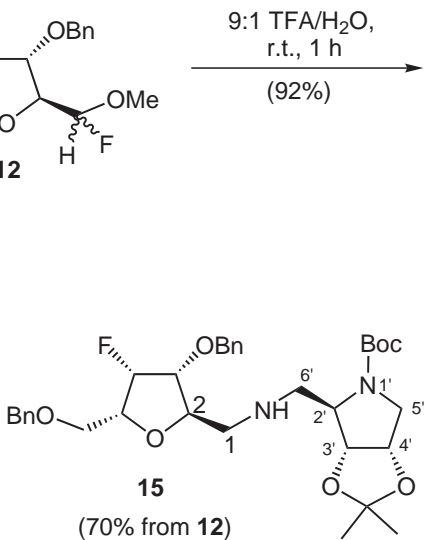

(70\% from 12)

Scheme 3

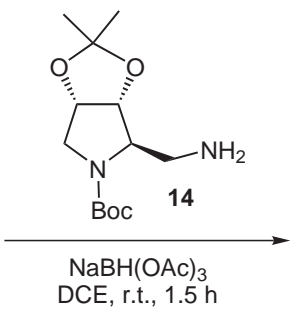

DCE, r.t., $1.5 \mathrm{~h}$

\section{References and Notes}

(1) (a) Fischer, C.; Lipata, F.; Rohr, J. J. Am. Chem. Soc. 2003, 125, 7818. (b) Faizi, S.; Ali, H. Planta Med. 1999, 65, 383.

(2) (a) Gremyachinskiy, D. E.; Samoshin, V. V.; Gross, P. H. Tetrahedron Lett. 2003, 44, 6587. (b) Bhat, A. S.; GervayHague, J. Org. Lett. 2001, 3, 2081. (c) BeMiller, J. N.; Gilson, R. J.; Myers, R. W.; Santoro, M. M.; Yadav, M. P. Carbohydr. Res. 1993, 250, 93. (d) Lai, W.; Martin, O. R. Carbohydr. Res. 1993, 250, 185. (e) Maity, S. K.; Dutta, S. K.; Banerjee, A. K.; Achari, B.; Singh, M. Tetrahedron 1994, 50, 6965. For 2-(aminomethyl)pyrrolidine-3,4-diol derivatives, see: (f) Saotome, C.; Wong, C.-H.; Kanie, O. Chem. Biol. 2001, 8, 1061. (g) Popowycz, F.; GerberLemaire, S.; Schütz, C.; Vogel, P. Helv. Chim. Acta 2004, 87, 800. (h) Fiaux, H.; Popowycz, F.; Favre, S.; Schütz, C.; Vogel, P.; Gerber-Lemaire, S.; Juillerat-Jeanneret, L. J. Med. Chem. 2005, 48, 4237. (i) Popowycz, F.; GerberLemaire, S.; Rodriguez-García, E.; Schütz, C.; Vogel, P. Helv. Chim. Acta 2003, 86, 1914.

(3) (a) Gruner, S. A. W.; Locardi, E.; Lohof, E.; Kessler, H. Chem. Rev. 2002, 102, 491. (b) Durrat, F.; Xie, J.; Valéry, J.-M. Tetrahedron Lett. 2004, 45, 1477.

(4) For recent reviews see: (a) Chakraborty, T. K.; Srinivasu, P.; Tapadar, S.; Mohan, B. K. Glycoconjugate J. 2005, 22, 83. (b) Chakraborty, T. K.; Srinivasu, P.; Tapadar, S.; Mohan, B. J. Chem. Sci. 2004, 116, 187. See also: (c) Prasad, S.; Mathur, A.; Jaggi, M.; Sharma, R.; Gupta, N.; Reddy, V. R.; Sudhakar, G.; Kumar, S. U.; Kumar, S. K.; Kunwar, A. C.; Chakraborty, T. K. J. Pept. Res. 2005, 66, 75. (d) Chakraborty, T. K.; Jayaprakash, S.; Diwan, P. V.; Nagaraj, R.; Jampani, S. R. B.; Kunwar, A. C. J. Am. Chem. Soc. 1998, 120, 12962. (e) Chakraborty, T. K.; Ghosh, S.; Jayaprakash, S.; Sarma, J. A. R. P.; Ravikanth, V.; Diwan, P. V.; Nagaraj, R.; Kunwar, A. C. J. Org. Chem. 2000, 65, 6441.

(5) Patrick, G. L. An Introduction to Medicinal Chemistry, 2nd ed.; Oxford University Press: Oxford, 2002, 446.

(6) (a) Broadley, K. J.; Kelly, D. R. Molecules 2001, 6, 142. (b) Liu, J.-K. Chem. Rev. 2005, 105, 2723.

(7) (a) Locardi, E.; Stöke, M.; Gruner, S.; Kessler, H. J. Am. Chem. Soc. 2001, 123, 8189. (b) Graf von Roedern, E.; Kessler, H. Angew. Chem., Int. Ed. Engl. 1994, 33, 687. (c) Graf von Roedern, E.; Kessler, H. Angew. Chem., Int. Ed. Engl. 1994, 33, 684.

(8) Benksim, A.; Beaupère, D.; Wadouahi, A. Org. Lett. 2004, 6, 3913.

(9) Mantell, S. J.; Fleet, G. W. J.; Brown, D. J. Chem. Soc., Perkin Trans. 1 1992, 3023. 
(10) (a) Vera-Ayoso, Y.; Borrachero, P.; Cabrera-Escribano, F.; Carmona, A. T.; Gómez-Guillén, M. Tetrahedron: Asymmetry 2004, 15, 429. (b) Borrachero, P.; CabreraEscribano, F.; Carmona, A. T.; Gómez-Guillén, M. Tetrahedron: Asymmetry 2000, 11, 2927. (c) BorracheroMoya, P.; Cabrera-Escribano, F.; Gómez-Guillén, M.; Madrid-Díaz, F. Tetrahedron Lett. 1997, 38, 1231.

(11) Vera-Ayoso, Y.; Borrachero, P.; Cabrera-Escribano, F.; Gómez-Guillén, M. Tetrahedron: Asymmetry 2005, 16, 889.

(12) General Procedure for the One-Pot Preparation of Compounds 7.

Compound 6 (100 mg, $0.315 \mathrm{mmol})$ was dissolved in a 9:1 TFA- $\mathrm{H}_{2} \mathrm{O}$ mixture $(2.7 \mathrm{~mL})$ and the solution was kept at r.t. for $1 \mathrm{~h}$. The reaction mixture was poured into ice-water (100 $\mathrm{mL})$ and extracted with $\mathrm{CH}_{2} \mathrm{Cl}_{2}(4 \times 20 \mathrm{~mL})$. The combined organic layers were successively washed with sat. aq $\mathrm{NaHCO}_{3}$ and brine, then dried $\left(\mathrm{Na}_{2} \mathrm{SO}_{4}\right)$, and concentrated. The residue (crude aldehyde) was dissolved in 1,2dicloroethane $(3.1 \mathrm{~mL})$ and treated with the amine $(0.437$ $\mathrm{mmol})$ and sodium triacetoxyborohydride $(93.0 \mathrm{mg}, 0.441$ mmol). The reaction was stirred at r.t. for the appropriate time (Table 1). The mixture was then diluted with sat. aq $\mathrm{NaHCO}_{3}(25 \mathrm{~mL})$ and the aqueous layer was extracted with EtOAc $(3 \times 20 \mathrm{~mL})$. The combined organic layers were dried $\left(\mathrm{Na}_{2} \mathrm{SO}_{4}\right)$, and concentrated under reduced pressure to give the crude product, which was purified by column chromatography using EtOAc-hexane, $\mathrm{Et}_{2} \mathrm{O}$-hexane or $\mathrm{Et}_{2} \mathrm{O}-$ acetone as eluent.

Compound 7a: $R_{f}=0.37\left(5: 1 \mathrm{Et}_{2} \mathrm{O}-\right.$ acetone $) ;[\alpha]_{\mathrm{D}}{ }^{26}+19.3(c$ $0.56, \mathrm{CH}_{2} \mathrm{Cl}_{2}$ ). IR: $v_{\max }=3324(\mathrm{NH}), 2106\left(\mathrm{~N}_{3}\right), 1746(\mathrm{CO})$, 1231 and $1119(\mathrm{CO}) \mathrm{cm}^{-1} .{ }^{1} \mathrm{H}$ NMR $\left(300 \mathrm{MHz}\right.$, acetone- $\left.d_{6}\right)$ : $\delta=7.25-7.08(\mathrm{~m}, 5 \mathrm{H}, \mathrm{Ph}), 5.31\left(\mathrm{dd}, 1 \mathrm{H}, J_{4,5}=7.8 \mathrm{~Hz}\right.$,

$\left.J_{3,4}=5.1 \mathrm{~Hz}, \mathrm{H}-4\right), 4.48\left(\mathrm{dd}, 1 \mathrm{H}, J_{2,3}=3.9 \mathrm{~Hz}, \mathrm{H}-3\right), 4.32$ (ddd, $\left.1 \mathrm{H}, J_{1,2}=J_{1^{\prime}, 2}=6.6 \mathrm{~Hz}, \mathrm{H}-2\right), 4.23(\mathrm{dd}, 1 \mathrm{H}$, $\left.J_{6,6^{\prime}}=10.8 \mathrm{~Hz}, J_{5,6}=2.4 \mathrm{~Hz}, \mathrm{H}-6\right), 4.07-3.95(\mathrm{~m}, 2 \mathrm{H}, \mathrm{H}-5$ and $\left.\mathrm{H}^{\prime} 6^{\prime}\right), 3.83\left(\mathrm{~d}, 1 \mathrm{H}, J_{\mathrm{H}, \mathrm{H}^{\prime}}=13.5 \mathrm{~Hz}, \mathrm{CH}^{a} \mathrm{Ph}\right), 3.78(\mathrm{~d}, 1$ $\left.\mathrm{H}, J_{\mathrm{H}, \mathrm{H}^{\prime}}=13.8 \mathrm{~Hz}, \mathrm{CH}{ }^{b} \mathrm{Ph}\right), 2.82\left(\mathrm{~d}, 2 \mathrm{H}, \mathrm{H}-1\right.$ and $\left.\mathrm{H}-1^{\prime}\right), 2.11$ and 2.02 (each $2 \mathrm{~s}, 3 \mathrm{H}, 2 \mathrm{COMe}$ ) ppm. ${ }^{13} \mathrm{C}$ NMR $(75.4 \mathrm{MHz}$, acetone- $\left.d_{6}\right): \delta=170.8,170.7$ (2 CO), $141.8-127.5(\mathrm{Ph}), 80.0$ (C-2), 78.1 (C-5), 75.8 (C-4), 64.6 (C-6), 64.4 (C-3), 54.4 $\left(\mathrm{CH}_{2} \mathrm{Ph}\right), 49.4(\mathrm{C}-1), 20.7$ and $20.4(2 \mathrm{COMe}) \mathrm{ppm}$. HRMS (CI): $\mathrm{m} / z$ calcd for $\mathrm{C}_{17} \mathrm{H}_{22} \mathrm{~N}_{4} \mathrm{O}_{5}+\mathrm{H}$ : 363.1668; found 363.1671.

(13) General Procedure for Deacetylation of 7 and Preparation of Compounds 8.

The corresponding reductive amination product $7(0.070$ mmol) was dissolved in: (i) ( $2 \mathrm{~mL}$ of $1: 1 \mathrm{MeOH}-\mathrm{CHCl}_{3}$ ), (ii) ( $2 \mathrm{~mL}$ of $\mathrm{MeOH}$ ), or (iii) (2 $\mathrm{mL}$ of EtOH abs.), and 5 drops of $1 \mathrm{M} \mathrm{MeONa}-\mathrm{MeOH}$ were added to the solution [for the deprotection of $\mathbf{7 g}$ was used EtONa-EtOH abs. (1 M)]. The reaction mixture was kept at r.t. for $2 \mathrm{~h}$. Work-up was done by one of the following procedures.

Procedure $1(\mathbf{8 b}-\mathbf{d}, \mathbf{f})$ : the reaction mixture was cooled and $600 \mu \mathrm{L}$ TFA was added. The residue was purified by a Dowex $50 \times 8 \mathrm{~W}$ column, using $\mathrm{MeOH}(50 \mathrm{~mL}), \mathrm{H}_{2} \mathrm{O}(50$ $\mathrm{mL})$ and $\mathrm{NH}_{4} \mathrm{OH}(10 \%$ aq soln; $100 \mathrm{~mL})$ as eluents.

Procedure $2(\mathbf{8 a}, \mathbf{e}, \mathbf{g}, \mathbf{h})$ : the reaction mixture was neutralized with Amberlyst 15, the resin was removed by filtration and the solvent under reduced pressure.
(14) In comparison with the NMR spectra of each direct precursor, each acetylated compound $\mathbf{7 a}-\mathbf{h}$ lacked any signal of aldehyde proton and carbon, but showed instead the signals corresponding to the two new diastereotopic protons at $\mathrm{C}(1)$. For the compounds obtained from some primary amines $(\mathbf{7 e}, \mathbf{f}-\mathbf{h})$, the amine proton gave rise to the typical broad signal in the ${ }^{1} \mathrm{H}$ NMR spectrum at $\delta=4.41$ (7e), 4.444.38 (7f), $5.81(\mathbf{7 g})$, and $6.00 \mathrm{ppm}(\mathbf{7 h})$, values that can be correlated with the electron-withdrawing or electrondonating character of the substituent at the para position of the aromatic group. However, the amine proton signal of $7 \mathbf{a}$ was not observed, probably because it is overlapped. The molecular weight found for 9 in its HRMS agreed with the aldimine structure assigned, while its NMR spectra showed the $s p^{2}(\mathrm{C}) \mathrm{H}$ signal at $\delta=6.86 \mathrm{ppm}$ and the imine carbon at $\delta=134.1 \mathrm{ppm}$, thus corroborating the assignation. For the deacetylated compounds 8a-h, their respective calculated molecular weights were in agreement with those found by HRMS. Furthermore, the ${ }^{1} \mathrm{H}$ NMR and ${ }^{13} \mathrm{C}$ NMR spectra of these compounds showed no signal corresponding to the $O$ acetyl groups present in the precursors $\mathbf{7 a}-\mathbf{h}$, as expected.

(15) Popowycz, F.; Gerber-Lemarie, S.; Demange, R.; Rodriguez-García, E.; Asenjo, A. T. C.; Robina, I.; Vogel, P. Bioorg. Med. Chem. Lett. 2001, 11, 2489.

(16) Compound 15 was obtained from 12 (100 mg, $0.265 \mathrm{mmol})$ and diamine $14(78 \mathrm{mg}, 0.287 \mathrm{mmol})$ in the presence of $\mathrm{NaBH}(\mathrm{OAc})_{3}(60.2 \mathrm{mg}, 0.287 \mathrm{mmol})$ by a similar one-pot procedure to that described above for the preparation of compounds 7 from 6.

More relevant data of 15: $R_{f}=0.45\left(\mathrm{Et}_{2} \mathrm{O}\right) ;[\alpha]_{\mathrm{D}}{ }^{24}+14.6(c$ 0.63 , acetone). IR: $v_{\max }=3295(\mathrm{NH}), 1692(\mathrm{CO}), 1370$ (NCO), 1157, 1059 (COC), and $991(\mathrm{CF}) \mathrm{cm}^{-1} .{ }^{1} \mathrm{H} \mathrm{NMR}$ $\left(500 \mathrm{MHz}, \mathrm{DMSO}-d_{6}, 363 \mathrm{~K}\right): \delta=7.41-7.25(\mathrm{~m}, 5 \mathrm{H}, \mathrm{Ph})$, $5.27\left(\mathrm{dt}, 1 \mathrm{H},{ }^{2} J_{4, \mathrm{~F}}=54.9 \mathrm{~Hz}, J_{3,4}=J_{4.5}=3.0 \mathrm{~Hz}, \mathrm{H}-4\right), 4.79$, $4.63\left(2 \mathrm{~d}, 1 \mathrm{H}\right.$ each, $\left.J_{\mathrm{H}, \mathrm{H}^{\prime}}=11.5 \mathrm{~Hz}, \mathrm{CH}_{2} \mathrm{Ph}\right), 4.69(\mathrm{dd}, 1 \mathrm{H}$, $\left.J_{4^{\prime}, 3^{\prime}}=J_{4^{\prime}, 5^{\prime} \mathrm{b}}=5.7 \mathrm{~Hz}, \mathrm{H}-4^{\prime}\right), 4.62\left(\mathrm{~d}, 1 \mathrm{H}, \mathrm{H}-3^{\prime}\right), 4.55(\mathrm{~s}, 2 \mathrm{H}$, $\mathrm{CH}_{2} \mathrm{Ph}$ ), 4.38 (dddd, $1 \mathrm{H},{ }^{3} \mathrm{~J}_{5, \mathrm{~F}}=30.5 \mathrm{~Hz}, J_{5,6 \mathrm{a}}=J_{5,6 \mathrm{~b}}=6.0$ $\mathrm{Hz}, \mathrm{H}-5), 4.33-4.21$ (m, $2 \mathrm{H}, \mathrm{H}-2$ and H-2'), 4.17 (dt, $1 \mathrm{H}$, $\left.{ }^{3} J_{3, \mathrm{~F}}=23.5 \mathrm{~Hz}, J_{2,3}=8.5 \mathrm{~Hz}, \mathrm{H}-3\right), 3.75(\mathrm{dd}, 1 \mathrm{H}$, $\left.J_{6 \mathrm{a}, 6 \mathrm{~b}}=10.2 \mathrm{~Hz}, \mathrm{H}-6 \mathrm{a}\right), 3.75\left(\mathrm{~d}, 1 \mathrm{H}, J_{5^{\prime} \mathrm{a}, 5^{\prime} \mathrm{b}}=14.0 \mathrm{~Hz}, \mathrm{H}-5^{\prime} \mathrm{a}\right)$, 3.60 (ddd, $\left.1 \mathrm{H},{ }^{4} J_{6 \mathrm{~b}, \mathrm{~F}}=1.8 \mathrm{~Hz}, \mathrm{H}-6 \mathrm{~b}\right), 3.32$ (dd, $1 \mathrm{H}, \mathrm{H}-5^{\prime} \mathrm{b}$ ), 3.20-2.91 (m, 4 H, H-1a, H-1b, H-6'a, H-6'b), 1.41 (s, 9 H, $\mathrm{CMe}_{3}$ ), 1.34 and 1.25 (each $2 \mathrm{~s}, 3 \mathrm{H}, \mathrm{CMe} e_{2}$ ) ppm. ${ }^{13} \mathrm{C} \mathrm{NMR}$ $\left(125.7 \mathrm{MHz}, \mathrm{DMSO}-d_{6}, 363 \mathrm{~K}\right): \delta=152(\mathrm{CO}), 137.7-126.8$ $(\mathrm{Ph}), 110.6\left(\mathrm{CMe}_{2}\right), 89.1\left(\mathrm{~d},{ }^{1} J_{4, \mathrm{~F}}=188.2 \mathrm{~Hz}, \mathrm{C}-4\right), 81.6(\mathrm{C}-$ $\left.3^{\prime}\right), 80.1\left(\mathrm{~d},{ }^{2} J_{3, \mathrm{~F}}=16.2 \mathrm{~Hz}, \mathrm{C}-3\right), 79.5\left(\mathrm{C}-4^{\prime}\right), 78.4(\mathrm{~d}$, $\left.{ }^{2} J_{5, \mathrm{~F}}=17.1 \mathrm{~Hz}, \mathrm{C}-5\right), 78.3\left(\mathrm{CMe}_{3}\right), 74.7(\mathrm{C}-2), 72.2$ and 71.2 $\left(\mathrm{CH}_{2} \mathrm{Ph}\right), 67.0\left(\mathrm{~d},{ }^{3} \mathrm{~J}_{6, \mathrm{~F}}=11.6 \mathrm{~Hz}, \mathrm{C}-6\right), 59.8\left(\mathrm{C}-2^{\prime}\right), 50.4(\mathrm{C}-$ $\left.5^{\prime}\right), 49.0,46.6\left(\mathrm{C}-1, \mathrm{C}-6^{\prime}\right), 27.6\left(\mathrm{CMe}_{3}\right), 26.3$ and 26.2 $\left(\mathrm{CMe} e_{2}\right.$ ). HRMS (CI): $\mathrm{m} / z$ calcd for $\mathrm{C}_{33} \mathrm{H}_{45} \mathrm{~N}_{2} \mathrm{O}_{7} \mathrm{~F}+\mathrm{H}$ : 601.3289; found: 601.3281 . 